\title{
Differentiation of Linear and Cyclic Polymer Architectures by MALDI Tandem Mass Spectrometry (MALDI-MS ${ }^{2}$ )
}

\author{
Aleer M. Yol, ${ }^{1}$ David E. Dabney, ${ }^{1,4}$ Shih-Fan Wang, ${ }^{2}$ Boyd A. Laurent, ${ }^{3}$ Mark D. Foster, ${ }^{2}$ \\ Roderic P. Quirk, ${ }^{2}$ Scott M. Grayson, ${ }^{3}$ Chrys Wesdemiotis ${ }^{1,2}$ \\ ${ }^{1}$ Department of Chemistry, The University of Akron, Akron, OH 44325, USA \\ ${ }^{2}$ Department of Polymer Science, The University of Akron, Akron, OH 44325, USA \\ ${ }^{3}$ Department of Chemistry, Tulane University, New Orleans, LA 70118, USA \\ ${ }^{4}$ Stepan Company, 22 W. Frontage Road, Northfield, IL 60093, USA
}

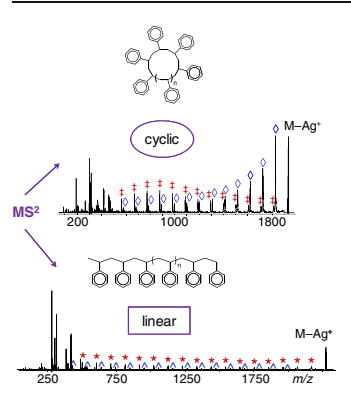

\begin{abstract}
M}+\mathrm{Ag}]^{+}$ions from cyclic and linear polystyrenes and polybutadienes, formed by matrix-assisted laser desorption ionization (MALDI), give rise to significantly different fragmentation patterns in tandem mass spectrometry $\left(\mathrm{MS}^{2}\right)$ experiments. In both cases, fragmentation starts with homolytic cleavage at the weakest bond, usually a $\mathrm{C}-\mathrm{C}$ bond, to generate two radicals. From linear structures, the separated radicals depolymerize extensively by monomer losses and backbiting rearrangements, leading to low-mass radical ions and much less abundant medium- and high-mass closed-shell fragments that contain one of the original end groups, along with internal fragments. With cyclic structures, depolymerization is less efficient, as it can readily be terminated by intramolecular
\end{abstract} $\mathrm{H}$-atom transfer between the still interconnected radical sites (disproportionation). These differences in fragmentation reactivity result in substantially different fragment ion distributions in the $\mathrm{MS}^{2}$ spectra. Simple inspection of the relative intensities of low- versus high-mass fragments permits conclusive determination of the macromolecular architecture, while full spectral interpretation reveals the individual end groups of linear polymers or the identity of the linker used to form the cyclic polymer.

Key words: Synthetic polymers, Macromolecular architecture, Cyclic versus linear structure, Tandem mass spectrometry, MALDI

Received: 10 May 2012/Revised: 4 September 2012/Accepted: 5 September 2012/Published online: 29 November 2012

\section{Introduction}

Synthetic polymers with unique structures, which endow $S$ them with specific chemical and physical properties, are widely used in the manufacture of industrial, consumer, and biomedical products. The development of such materials is, therefore, a major task in polymer science [1]. Cyclic polymers have gained attention in the polymer community because their lack of end groups leads to reduced hydrodynamic volumes, resulting in distinctive adhesion, viscoelastic, and dispersion properties [2]. Polymers with macrocyclic architectures can be efficiently prepared by cyclization of properly substituted $\alpha, \omega$ -

Electronic supplementary material The online version of this article (doi:10.1007/s13361-012-0497-5) contains supplementary material, which is available to authorized users.

Correspondence to: Chrys Wesdemiotis; e-mail: wesdemiotis@uakron.edu difunctional chains [3-8]. Unequivocal characterization of the macrocyclic architectures has been challenging, however, because isomeric linear structures, terminated by a double bond, are possible. Linear and cyclic polymeric isomers have been distinguished by ion mobility mass spectrometry [9]. Here, we evaluate the applicability of tandem (2-D) mass spectrometry $\left(\mathrm{MS}^{2}\right)$ for differentiating cyclic from linear polymers.

$\mathrm{MS}^{2}$ studies have been reported for linear, branched, and dendritic polymer systems [10-39]. The present investigation applies $\mathrm{MS}^{2}$ to macrocyclic polystyrenes with welldefined connectivity, prepared by living anionic polymerization and metathesis ring-closure [8] and ionized by matrixassisted laser desorption ionization (MALDI) [40, 41]. The linear starting material, a $\alpha, \omega$-divinyl polystyrene, is also examined for comparison. From the distinctively different fragmentation pathways of linear and cyclic species, spectral interpretation rules for the assignment of architectures are devised [42] and tested with a cyclic polystyrene prepared 
by a different method, viz. click cyclization $[4,6]$. Finally, the $\mathrm{MS}^{2}$ characteristics of macrocyclic polybutadiene are briefly evaluated to determine whether $\mathrm{MS}^{2}$ fragmentation patterns offer a general means for establishing polyolefin architectures.

\section{Experimental}

MS and $\mathrm{MS}^{2}$ experiments were performed on a Bruker UltraFlex III MALDI tandem time-of-flight (TOF/TOF) mass spectrometer (Bruker Daltonics, Billerica, MA, USA) equipped with a Nd:YAG laser emitting at $355 \mathrm{~nm}$ [43]. DCTB, viz. \{2-[(2E)-3-(4-tert-butylphenyl)-2-methylprop-2enylidene]malonitrile\} ( $\geq 98 \%$; Santa Cruz Biotechnology, Santa Cruz, CA, USA), and silver trifluoroacetate (98\%; Aldrich, Milwaukee, WI, USA) served as matrix and cationization salt, respectively. Solutions of the matrix $(20 \mathrm{mg} / \mathrm{mL})$, cationizing salt $(10 \mathrm{mg} / \mathrm{mL})$, and polymer sample $(10 \mathrm{mg} / \mathrm{mL})$ were prepared in THF $(99.9 \%$; Aldrich). The matrix/polymer/cationizing agent solutions were mixed in the ratio 10:2:1 ( $\mathrm{vol} / \mathrm{vol} / \mathrm{vol})$, and $0.5-1.0 \mu \mathrm{L}$ of the final mixture were applied to the MALDI sample target and allowed to dry at ambient conditions before spectral acquisition. This sample preparation protocol led to the formation of $[\mathrm{M}+\mathrm{Ag}]^{+}$ions and, from labile polymers, also their fragments.

The UltraFlex III TOF/TOF mass spectrometer is composed of a short linear TOF tube, interfaced axially with a reflectron TOF device [43, 44]. $\mathrm{MS}^{2}$ spectra were acquired using the LIFT technique, which involves raising the laser intensity to induce fragmentation in the linear TOF part, isolation of the desired precursor ion and its fragments ("ion family") by a high-resolution timed ion selector (TIS), and post-acceleration ("lifting") of the selected ion family for mass analysis in the reflectron TOF segment. The IS1, IS2, and lens potentials in the ion source were set at 8.00,7.15, and $3.60 \mathrm{kV}$, respectively, the LIFT 1 and 2 potentials at 19.00 and $2.90 \mathrm{kV}$, respectively, and the reflectron 1 and 2 lenses at 29.50 and $13.85 \mathrm{kV}$. For single-stage (1-D) mass spectra, the TIS and LIFT devices were grounded, and the IS1, IS2, source lens, reflectron 1 , and reflectron 2 potentials were set at $25.03,21.72,9.65,26.32$, and $13.73 \mathrm{kV}$, respectively.

The synthetic routes to the samples analyzed have been described in detail elsewhere $[4,8,45,46]$. Briefly, $\alpha, \omega-$ difunctional polystyrenes $[4,6,8,45]$ were prepared by living anionic polymerization $[8,45]$ or atom transfer radical polymerization $[4,6]$ and cyclized by metathesis ringclosure $[8,45]$ or click chemistry $[4,6]$. Linear and cyclic polybutadienes were prepared by conventional living anionic polymerization of butadiene [46] and ring-opening metathesis polymerization of 1,5,9-cyclododecatriene [46, 47], respectively. All products were isolated by precipitation and vacuum filtration and their molecular structures were confirmed by ${ }^{1} \mathrm{H}$ and ${ }^{13} \mathrm{C}$ NMR spectroscopy and MALDITOF mass spectrometry $[4,8,46]$.

\section{Results and Discussion}

\section{Linear Polystyrene}

Living anionic polymerization of styrene using 4-pentenyllithium as initiator and $p$-vinylbenzyl chloride as the terminating electrophile gives rise to a telechelic polystyrene (PS) that carries 4-pentenyl and $p$-vinylbenzyl substituents at the $\alpha$ and $\omega$ chain ends, respectively (see structure in Figure 1 and Supplemental Scheme S1) [8, 45]. Reaction of $1.74 \mathrm{mmol}$ initiator with $41.5 \mathrm{mmol}$ monomer and $17.4 \mathrm{mmol}$ terminating agent led to a polymer with an average molecular weight $\left(\mathrm{M}_{\mathrm{n}}\right)$ of $2800 \pm 140 \mathrm{Da}$, as determined by size exclusion chromatography (SEC) with light scattering detection [45]. The corresponding MALDI mass spectrum (Supplemental Figure S1) shows a narrow molecular weight distribution in the desired molecular weight range and essentially one series of $[\mathrm{M}+\mathrm{Ag}]^{+}$ ions with the expected composition, in accord with the $\alpha$ pentenyl- $\omega$-vinylbenzyl chain end functionality needed for subsequent cyclization via metathesis ring-closure (vide infra).

Figure 1 shows the MALDI-MS ${ }^{2}$ spectrum of the silverated 19-mer of the divinyl-terminated polystyrene. This spectrum includes the typical fragments observed from the metal ion adducts of linear, chain-end functionalized polystyrenes, viz. abundant radical ions carrying either the $\alpha$ or the $\omega$ chain end $\left(b_{1}{ }^{\circ}, b_{2}{ }^{\circ}, z_{1}{ }^{\circ}\right)$ and internal ions $\left(J_{2}{ }^{\circ}, K_{3}\right)$ in the low-mass region (see Supplemental Scheme S2 for their structures), as well as two homologous series of relatively sizable fragments $\left(a_{n}, y_{n}\right)$ across the medium- and high-mass region, each containing one original end group and one newly produced methylene end group (Figure 2) [42]. This fragmentation pattern arises from random, charge-remote homolytic $\mathrm{C}-\mathrm{C}$ bond cleavages along the polymer backbone which create charged radicals that may decompose further by backbiting rearrangements (Supplemental Scheme S2), bond migrations (phenyl shifts), and $\beta$ scissions $[16,20,21]$.

Figure 2 explains the nomenclature used and summarizes the structures of the fragments generated from linear polystyrenes [42]. Initial $\mathrm{C}-\mathrm{C}$ bond breakup in the PS backbone yields truncated chains with benzylic $\left(b_{n}{ }^{\circ}, z_{n}{ }^{\circ}\right)$ and primary $\left(\mathrm{a}_{\mathrm{n}}{ }^{\circ}, \mathrm{y}_{\mathrm{n}}{ }^{\circ}\right)$ radical sites at the chain ends, which can undergo unzipping, occurring by consecutive monomer losses via $\beta \mathrm{C}-\mathrm{C}$ bond scissions, as well as loss of a hydrogen or phenyl radical to produce six different fragment ion series, viz. $a_{n}, a_{n a}, b_{n}, y_{n}, y_{n a}$, and $z_{n}[20,21,42]$ (Figure 2). Only very small benzylic radical ions survive intact $\left(\mathrm{b}_{1}{ }^{\circ}, \mathrm{b}_{2}{ }^{*}, \mathrm{~b}_{3}{ }^{\cdot}, \mathrm{z}_{1}{ }^{\cdot}, \mathrm{z}_{2}{ }^{*}\right.$ ), indicating extensive (for the benzylic) or complete (for the primary radicals) depolymerization by unzipping. The longer lifetimes of the benzylic radicals also permit the occurrence of hydrogen rearrangements that move the radical site to a more stable internal position (backbiting), cf. Supplemental Scheme S2; subsequent $\mathrm{C}-\mathrm{C}$ bond cleavages provide an alternative route to the terminal series $a_{n}$ and $y_{n}$ and coproduce the internal fragments $\mathrm{J}_{2}{ }^{\circ}$ and $\mathrm{K}_{3}[20,42]$. The high relative intensities of $\mathrm{J}_{2}{ }^{\circ}$ and $\mathrm{K}_{3}$ in the low-mass range of the $\mathrm{MS}^{2}$ spectrum and of the $a_{n} / y_{n}$ doublets in the medium- and high-mass ranges 




Figure 1. MALDI-MS ${ }^{2}$ spectrum of the silverated 19-mer of $\alpha$-4-pentenyl- $\omega$-( $p$-vinylbenzyl) polystyrene $(m / z 2270.5)$; the fragment nomenclature is explained in Figure 2. The expanded trace shows the types of fragments observed in the medium and upper mass range of the spectrum

identify backbiting as the major dissociation pathway of the incipient charged radicals emerging after the initial PS backbone breakup. It is noteworthy that the relative abundances of the $y_{n}$ series are lower than those of the $a_{n}$ series, consistent with facile elimination of the $\omega$ end group which is attached to the polymer through a bond connecting two benzylic $\mathrm{C}$ atoms [21].

\section{Cyclic Polystyrenes}

The $\alpha, \omega$-divinyl polystyrene was cyclized by metathesis ringclosure using the Grubb's catalyst bis(tricyclohexylphosphine)benzylidene ruthenium (IV) chloride [47]; the macrocycle resulting from this reaction is depicted in Figure 3a. The MALDI mass spectrum (Supplemental Figure S2a) shows only one narrow distribution of $[\mathrm{M}+\mathrm{Ag}]^{+}$ions with the desired composition. The silverated $n$-mers from the cyclic PS appear $28 \mathrm{~m} / \mathrm{z}$ units lower than the same $n$-mers from the linear precursor (cf. Supplemental Scheme S1), in agreement with the Grubbs metathesis mechanism. In a subsequent step, the double bond in the macrocycle was hydrogenated using (triphenylphosphine)rhodium(I) chloride (Wilkinson's catalyst) [45], cf. structure in Figure 3b. The MALDI mass spectrum of the hydrogenated polymer (Supplemental Figure $\mathrm{S} 2 \mathrm{~b})$ shows a monomodal distribution of $[\mathrm{M}+\mathrm{Ag}]^{+}$ions, which are observed $2 \mathrm{~m} / z$ units higher than the same $n$-mers from the original cyclization product, confirming that the double bond within the macrocycle was quantitatively reduced. SEC analysis of the unsaturated and reduced macrocycle with light scattering detection indicated average molecular weights

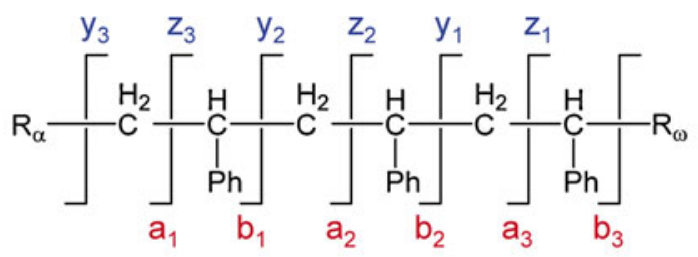<smiles>[X]C(=C)CC(C)(C(CC([R])(C)C)c1ccccc1)C([R20])(C)C</smiles><smiles>[R]C(C)(C)C(CC(C)(C)C(/C=C\c1ccccc1)c1ccccc1)Pc1ccccc1</smiles>

$$
\begin{array}{ll}
X=P h & a_{n} \\
X=H & a_{n a}
\end{array}
$$

$\mathrm{Z}_{\mathrm{n}}$

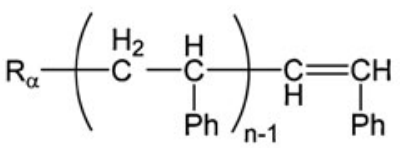

$\mathrm{b}_{\mathrm{n}}$

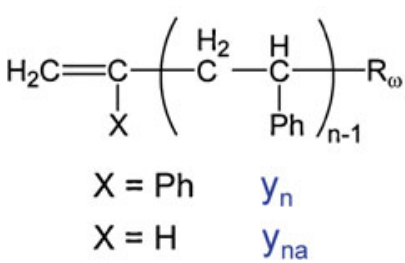

Figure 2. Nomenclature scheme for the $\mathrm{MS}^{2}$ fragments from linear polystyrenes and major terminal fragment ions; Ph symbolizes the phenyl substituent. The charge is provided by $\mathrm{Ag}^{+}$(omitted for brevity). Series $a_{n}$, $a_{n a}$, and $b_{n}$ contain the $\alpha$ end group $\left(R_{\alpha}\right)$ and series $y_{n}, y_{n a}$, and $z_{n}$ the $\omega$ end group $\left(R_{\omega}\right)$. The subscript indicates the number of complete or partial repeat units remaining in the fragment ion [20, 42] 


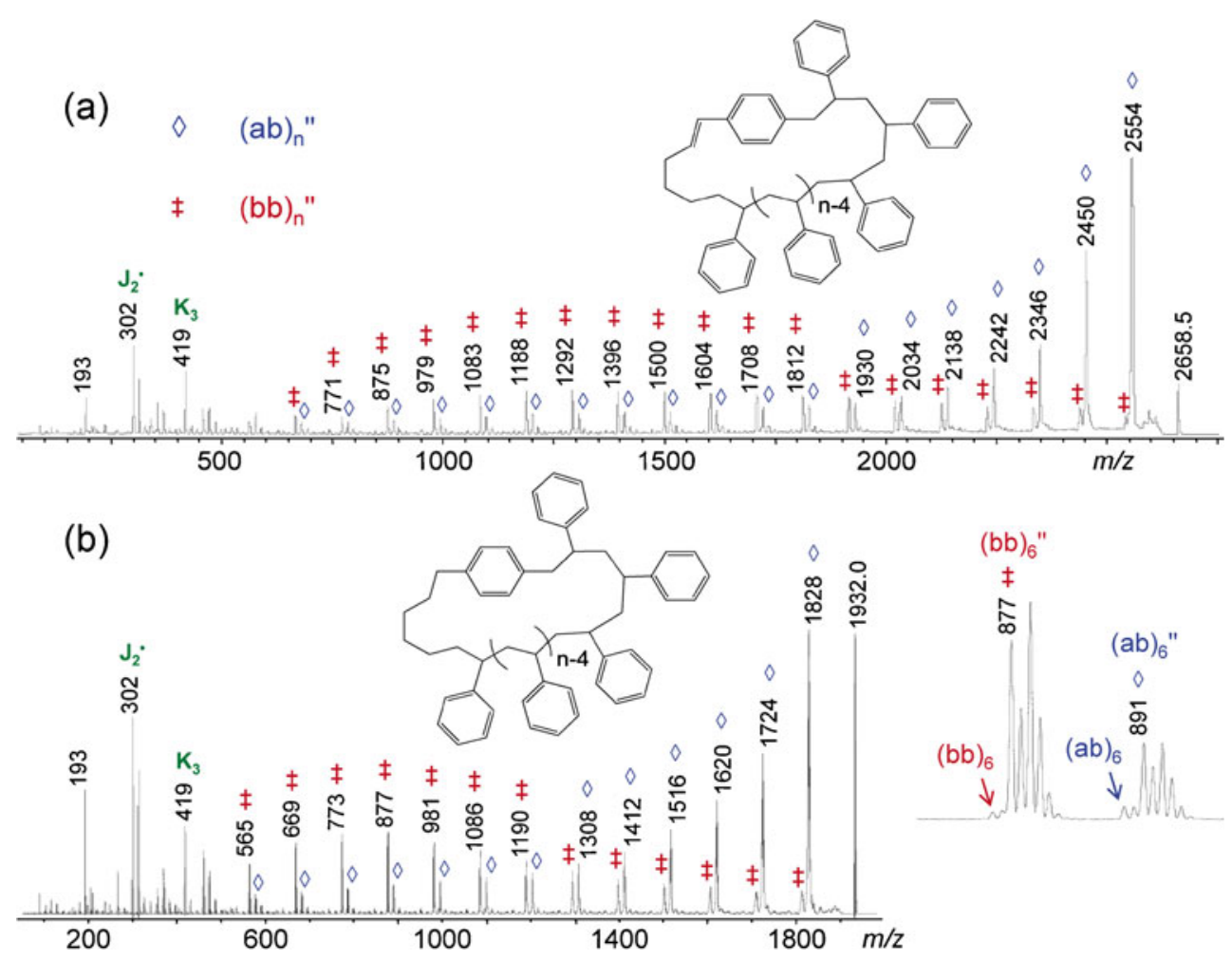

Figure 3. MALDI-MS ${ }^{2}$ spectra of (a) the silverated 23-mer from the macrocyclic PS obtained by metathesis ring-closure of $\alpha$ 4-pentenyl- $\omega$-( $p$-vinylbenzyl) polystyrene $(\mathrm{m} / z$ 2658.5) and (b) the silverated 16 -mer from the hydrogenated macrocycle $(\mathrm{m} / z$ 1932.0) and an expanded trace of the $m / z$ 870-900 region of this spectrum

$\left(\mathrm{M}_{\mathrm{n}}\right)$ of $2700 \pm 140$ and $2800 \pm 140 \mathrm{Da}$, respectively [8, 45]. Cyclization and hydrogenation do not change measurably the molecular weight distribution and average molecular weight, as is also evident from the corresponding MALDI mass spectra (cf. Supplemental Figures S1 and S2), and in accord with the chemical transformations effected in these reactions (cf. Supplemental Scheme S1).

The MALDI-MS ${ }^{2}$ spectrum of the silverated 23 -mer from the cyclic polystyrene obtained by metathesis ring-closure is markedly different from the $\mathrm{MS}^{2}$ spectrum of the linear precursor, cf. Figures 1 and $3 \mathrm{a}$. The linear $n$-mer preferentially yields fragments that are missing one end group $\left(a_{n} / y_{n}\right.$ pairs); in sharp contrast, the cyclic polystyrene mainly generates fragment ions by losses of $\mathrm{n}$ times the monomer, $\left(\mathrm{C}_{8} \mathrm{H}_{8}\right)_{\mathrm{n}}$, or $\mathrm{n}$ times the monomer plus an additional $\mathrm{CH}_{2}$ unit, which have been labeled by a blue $\diamond$ and a red $\$$ sign, respectively. Reducing the double bond in the macrocycle does not change this fragmentation pattern, as indicated by the MALDI-MS ${ }^{2}$ spectrum of the silverated 16-mer of the hydrogenated product, cf. Figure $3 \mathrm{~b}$. Essentially all fragments above $\mathrm{m} / z \sim 450$ in Figure $3 \mathrm{~b}$ appear $2 \mathrm{~m} / z$ units higher than the analogous fragments in Figure $3 \mathrm{a}$, providing strong evidence that they include the linker unit and that fragmentation occurs within the PS chain.

The energetically preferred dissociation within the PS chain is $\mathrm{CH}_{2}-\mathrm{CH}(\mathrm{Ph})$ bond cleavage. Such a reaction within the PS segment of $\mathrm{Ag}^{+}$-cationized macrocycles creates linear $\mathrm{Ag}^{+}$-cationized diradicals with one primary and one benzylic radical site at their termini, as shown in Scheme 1. Successive monomer losses (unzipping) are facile, especially at the primary radical site, based on the behavior of the radical ions formed from linear polystyrenes [20]. The unzipping process proceeds until stopped by intramolecular $\beta-\mathrm{H}^{*}$ transfer from one radical to the other, resulting in fragment ions with one saturated and one olefinic chain end ("disproportionation"). Scheme 1 shows $\mathrm{H}^{*}$ abstraction from the benzylic carbon, which should have a lower energy requirement than the alternative $\mathrm{H}^{*}$ transfer from a $\mathrm{CH}_{2}$ group. The fragment series formed this way has been termed $(\mathrm{ab})_{\mathrm{n}}{ }^{\prime \prime}$, because one chain end contains a methylene carbon atom (as in $a_{n}$ ), the other a benzylic carbon atom (as in $b_{n}$ ), and one of them is saturated (indicated by" [20,42].

The second major series is accounted for by a 1,2-phenyl migration (see Scheme 1) at the primary radical to form a more stable secondary radical that can lose phenylpropene $(118 \mathrm{Da})$ by $\beta \mathrm{C}-\mathrm{C}$ bond scission [21]. Phenyl propene contains one $\mathrm{CH}_{2}$ unit more than the monomer. A series of monomer losses may accompany this rearrangement, ultimately leading to a distribution of ring-opened diradical chains with benzylic radicals at both chains ends. Consecutive intramolecular $\beta-\mathrm{H}^{*}$ transfer between the radical sites is again possible to form fragment ions with one saturated and one olefinic chain end; these have been termed $(b b)_{n}{ }^{\prime \prime}$ fragments, as they carry benzylic carbons at both chain ends.

In addition to these major dissociation pathways, backbiting at the benzylic radicals takes place, as revealed by the 


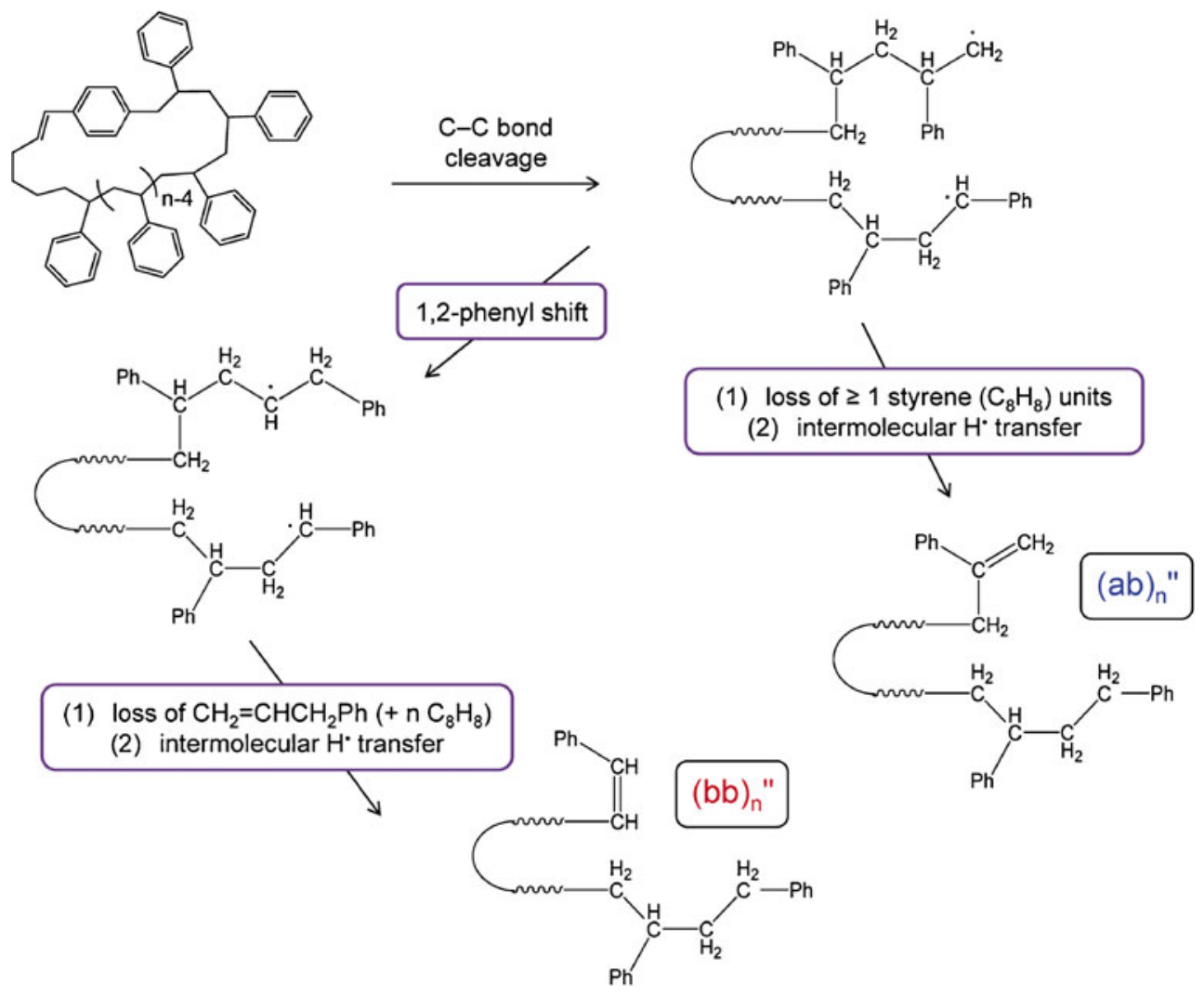

Scheme 1. Major dissociation pathways of silverated polystyrene macrocycles, commencing with random homolytic $\mathrm{C}-\mathrm{C}$ bond cleavages within the PS chain. The 1,2-phenyl shift may occur after ring opening or after one or more monomer units have been eliminated. All species are ionized by $\mathrm{Ag}^{+}$(omitted for brevity)

ions observed at $m / z 302\left(\mathrm{~J}_{2}{ }^{\circ}\right)$ and $419\left(\mathrm{~K}_{3}\right)$; the extent of this rearrangement is, however, significantly lower than in linear polystyrenes, cf. Figures 1 and 3. The $\mathrm{Ag}^{+}$-cationized diradicals arising after unzipping (or combined phenyl migration plus unzipping) could decompose by sequential $\beta$ - $\mathrm{H}^{*}$ elimination to produce fragments with two unsaturated chain ends, viz. series $(a b)_{n}$ and $(b b)_{n}$, which should appear $2 \mathrm{~m} / \mathrm{z}$ units lower than series (ab) ${ }_{\mathrm{n}}{ }^{\prime \prime}$ and (bb) ${ }_{\mathrm{n}}{ }^{\prime \prime}$, respectively. The expanded $\mathrm{MS}^{2}$ spectrum in Figure $3 b$ indeed confirms the occurrence of this process to a very small degree; the alternative, intramolecular $\mathrm{H}^{*}$ rearrangement is more com- petitive because it involves concomitant bond scission and bond formation, thus lowering the corresponding activation energy.

The foregoing discussion clearly shows that polymer architecture can influence the unimolecular chemistry taking place in $\mathrm{MS}^{2}$ experiments. Linear polystyrenes yield fragment ions that contain only one of the original end groups, whereas cyclic polystyrenes mainly decompose by monomer losses. Based on these characteristics, architectural differentiation by $\mathrm{MS}^{2}$ is possible. To test whether this approach is generally applicable, a PS macrocycle with

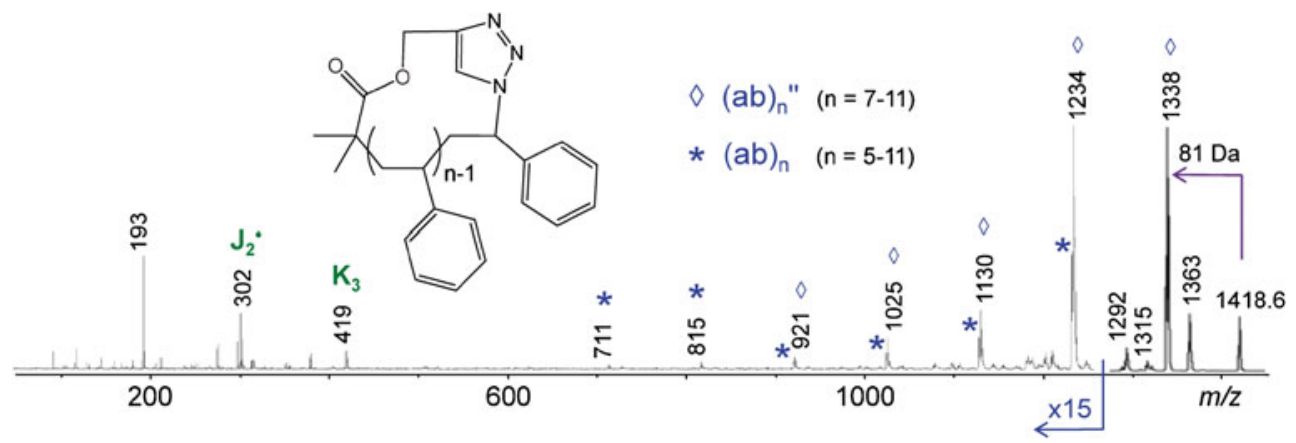

Figure 4. MALDI-MS ${ }^{2}$ spectrum of the silverated 11-mer $(\mathrm{m} / z$ 1418.6) from the macrocyclic PS obtained by click chemistry of $\alpha$-propargylisobutyryl- $\omega$-azido polystyrene (cf. Supplemental Supplemental Scheme S3). The relative intensity axis is expanded 15 times below $\mathrm{m} / \mathrm{z} 1260$ 
different linking connectivity was investigated (see structure in Figure 4).

The new polystyrene macrocycle was synthesized according to the four-step procedure summarized in Supplemental Scheme S3 [4]. First, atom transfer radical polymerization (ATPR) was utilized to form a linear polymer with propargyl isobutyrate and bromine substituents at the $\alpha$ and $\omega$ chain ends, respectively. Then, substitution of the bromine with an azide end group followed. In the final step, intramolecular azide-alkyne cycloaddition in the presence of $\mathrm{Cu}(\mathrm{I})$ catalyst (click chemistry) was performed to obtain a PS macrocycle that incorporates a linking unit containing both ester and triazole functionalities. The MALDI mass spectrum of the final product (Supplemental Figure S3) contains one Gaussian-shaped series of $[\mathrm{M}+\mathrm{Ag}]^{+}$ions with the expected composition, attesting the formation of a product with high purity and narrow molecular weight distribution $\left(\mathrm{M}_{\mathrm{n}}=1800\right.$ $\pm 90 \mathrm{Da}$ according to SEC).

Figure 4 depicts the MALDI-MS ${ }^{2}$ spectrum of the silverated 11-mer from the product of click cyclization. Extensive dissociation by loss of methylene triazole is observed $(m / z 1388)$, consistent with easy homolysis of the allylic $\mathrm{C}-\mathrm{O}$ and $\mathrm{C}-\mathrm{N}$ bonds in the linker unit introduced by click chemistry at the internal energies accessed under MALDI-MS ${ }^{2}$ conditions (cf. Scheme 2). These homolytic cleavages result in a silverated diradical containing a carboxy radical site, as well as a styryl radical site that can depolymerize (unzip); indeed, the fragment at $\mathrm{m} / \mathrm{z} 1388$ dissociates further by losses of $\mathrm{n}$ times styrene monomer, $\left(\mathrm{C}_{8} \mathrm{H}_{8}\right)_{\mathrm{n}}$, as did the diradicals arising from cyclic polystyrenes with a stable linker moiety (cf. Figures 3 and 4). Depolymerization proceeds until $\beta-\mathrm{H}^{*}$ abstraction from the terminal benzyl site by the carboxy radical generates closedshell ions, viz. series (ab)n" (cf. Scheme 2). The polystyrene chains of this series are terminated with one saturated methylene carbon atom (attached to the isobutyryl group) and one unsaturated benzylic carbon atom and, thus, have quite similar structural attributes to those from the macrocycles with a stable linker group (cf. Schemes 1 and 2).

Hydrogen atom abstraction from a $\mathrm{C}-\mathrm{H}$ bond by a carboxy radical is exothermic due to the high stability of the $\mathrm{COO}-\mathrm{H}$ bond $[48,49]$. In the population of fragmenting diradicals, in which the carboxy and benzyl sites are too far apart to permit $\beta-\mathrm{H}^{*}$ transfer between the chain ends, $\mathrm{H}^{*}$ rearrangement to the $\mathrm{COO}^{\circ}$ group can take place from the nearest (proximal) benzylic position through an energetically favored six-membered ring transition, as shown in Scheme 2; sequential $\beta-\mathrm{H}^{*}$ elimination from the two benzylic radicals generated in this process provides a plausible route to series $(\mathrm{ab})_{\mathrm{n}}$, which is observed $2 \mathrm{~m} / \mathrm{z}$ units lower than the major series $(a b)_{n}{ }^{\prime \prime}$.

It is noteworthy that the labile linker blocks the dissociation pathway to $(\mathrm{bb})_{\mathrm{n}}{ }^{\prime \prime}$ fragments because of selective ring-opening at the linking moiety. The ring-opened isomer lacks the primary radical site needed for a 1,2-phenyl shift, thereby obstructing $(\mathrm{bb})_{\mathrm{n}}{ }^{\prime \prime}$ formation.
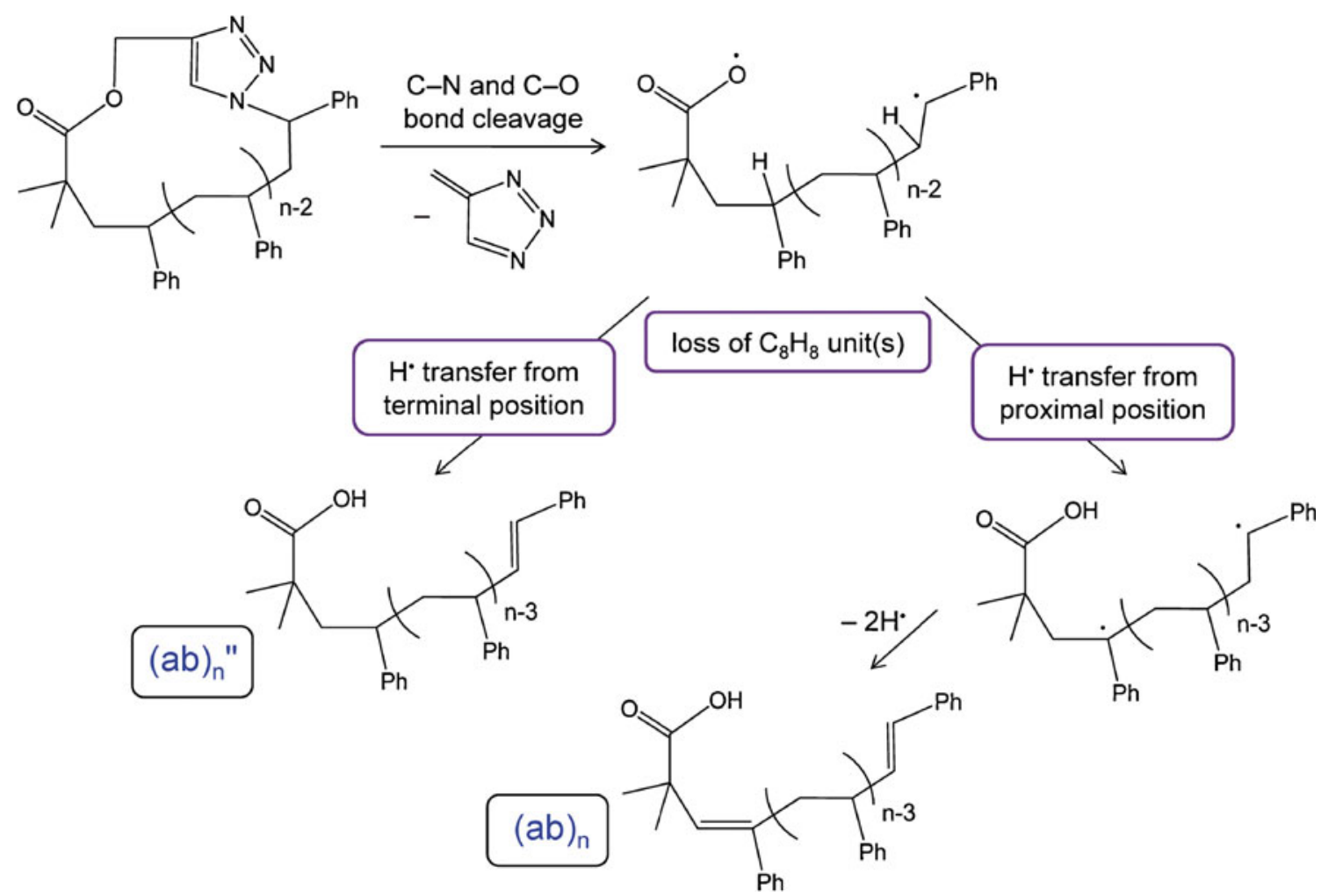

Scheme 2. Major dissociation pathways of silverated polystyrene macrocycles, commencing with selective homolytic bond cleavages within the linker substituent. All species are ionized by $\mathrm{Ag}^{+}$(omitted for brevity) 


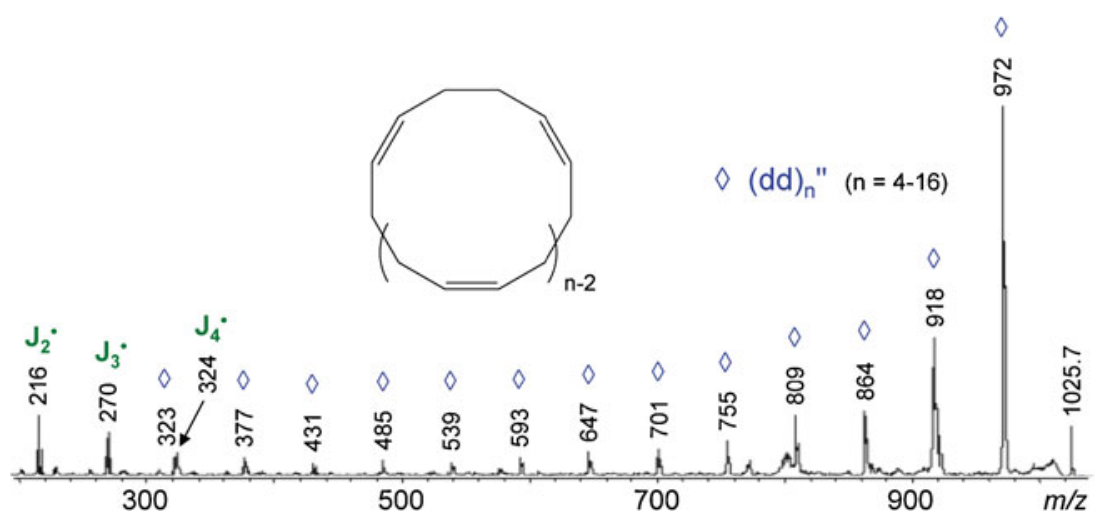

Figure 5. MALDI-MS ${ }^{2}$ spectrum of the silverated 17-mer $(\mathrm{m} / \mathrm{z} 1025.7)$ from a macrocyclic PB obtained by ring-opening metathesis polymerization of 1,5,9-cyclododecatriene [46]

Despite their structural and stability differences, the two classes of cyclic polystyrenes investigated undergo similar fragmentation chemistry. Both dissociate largely by unzipping, either directly from the $[\mathrm{M}+\mathrm{Ag}]^{+}$precursor ions, or after the loss of linking group elements. More importantly, abundant closedshell fragment ions in the upper mass region of the $\mathrm{MS}^{2}$ spectrum dominate, in contrast to linear structures, which dissociate to yield abundant open-shell (i.e. radical) ions in the lower mass region of the $\mathrm{MS}^{2}$ spectrum. These distinctive features allow for conclusive determination of the correct polymer architecture.

\section{Cyclic Polybutadiene}

Our results for polystyrene oligomers reveal that polymer architecture is an important determinant of the fragmentation patterns resulting from free radical chemistry. $\mathrm{MS}^{2}$ experiments on linear polybutadiene (PB) have shown that also this connectivity favors free radical chemistry when energetically activated [50]. Hence, cyclic PB was also examined in order to ascertain whether the preference for efficient unzipping by monomer losses is a general phenomenon of polymers undergoing unimolecular decay through homolytic bond cleavages and radical rearrangements.
The cyclic PB was prepared by ring-opening metathesis polymerization of 1,5,9-cyclododecatriene [46], which generated a polymer with the composition $\left(\mathrm{C}_{4} \mathrm{H}_{6}\right)_{n}$ and no additional functionality (see structure in Figure 5). The MALDI-MS ${ }^{2}$ spectrum of the silverated 17-mer from this product includes dominant fragments due to monomer losses (depolymerization) in the high and medium mass range along with radical ion fragments in the low mass range, $\mathrm{cf}$. Figure 5, as was encountered with the cyclic polystyrenes. More importantly, the $\mathrm{MS}^{2}$ spectrum of the macrocyclic oligomer is substantially different from the $\mathrm{MS}^{2}$ spectra reported for $\mathrm{Ag}^{+}$adducts of linear polybutadienes [50], which primarily decompose to low-mass radical ions that include one of the chain end groups (cf. Figure S4).

Scheme 3 provides a mechanistic rationalization for the major fragment ion series from the $\mathrm{PB}$ macrocycle. The weakest bond in the PB chain is the $\mathrm{CH}_{2}-\mathrm{CH}_{2}$ single bond $[48,51]$. Preferential dissociation at such a bond in the $\mathrm{Ag}^{+}-$ cationized 17-mer yields an isomeric linear, symmetric diradical with allylic radical sites at both chain ends, cf. Scheme 3. Monomer loss(es) from this intermediate and sequential intramolecular $\mathrm{H}$-atom transfer (disproportionation) between the radical sites leads to fragments with the nominal

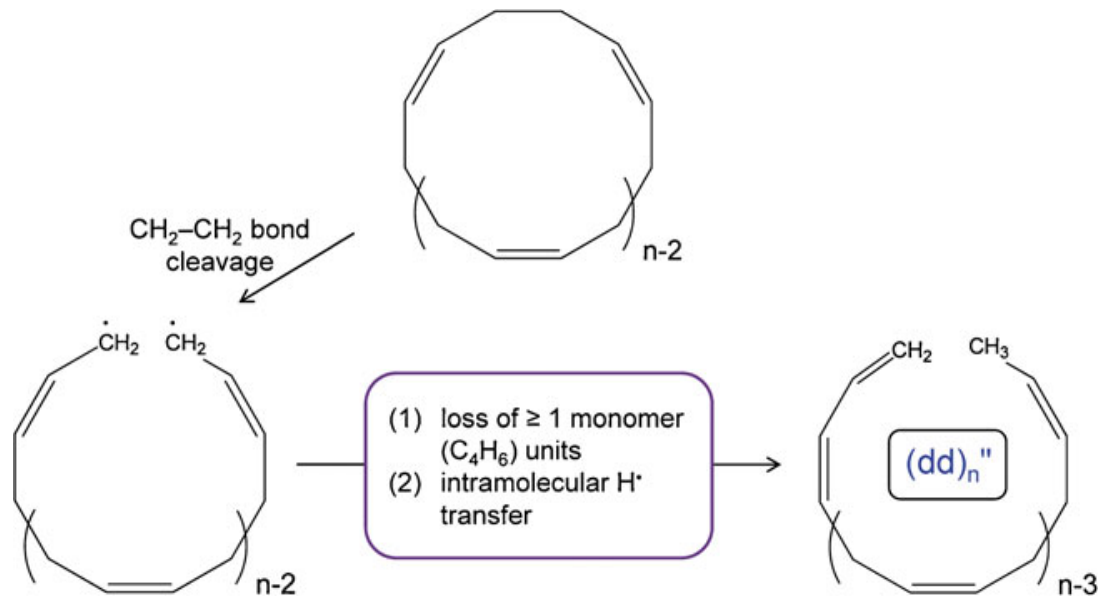

Scheme 3. Dissociation of silverated polybutadiene macrocycles via homolytic $\mathrm{CH}_{2}-\mathrm{CH}_{2}$ cleavages yielding two allylic radicals at the chain ends. All species are ionized by $\mathrm{Ag}^{+}$ 
composition $\left(\mathrm{C}_{4} \mathrm{H}_{6}\right)_{\mathrm{n}}$, bearing a truncated $\mathrm{PB}$ chain capped with $\mathrm{C}_{4} \mathrm{H}_{7}\left(\mathrm{CH}_{3} \mathrm{CH}=\mathrm{CHCH}_{2}-\right)$ and $\mathrm{C}_{4} \mathrm{H}_{5}\left(\mathrm{CH}_{2}=\mathrm{CHCH}=\mathrm{CH}-\right)$ end groups, as shown in Scheme 3. This series has been termed (dd) ${ }_{n}$ " because its chain ends are created by $\mathrm{C}-\mathrm{C}$ bond cleavage at the end of a complete repeat unit ("bond d," cf. Supplemental Figure S5) [42].

In the low mass region of Figure 5, a quite abundant radical ion series, $\mathrm{J}_{\mathrm{n}}{ }^{\circ}$, is detected. These low mass products are attributed to backbiting rearrangements in the allylic diradicals generated after ring opening and monomer loss(es). The observation of a series of radical ions further indicates that the migrating $\mathrm{H}^{*}$ may originate from a nearby location (as illustrated in Supplemental Scheme S2 for polystyrene) or from more distant locations down the chain. This behavior is consistent with the lower degree of crowding in PB, as compared to PS, which makes it easier for the reacting sites to approach each other for $\mathrm{H}^{*}$ rearrangement.

\section{Conclusions}

Linear and cyclic architectures of polystyrenes and polybutadienes show significantly different fragmentation characteristics in tandem mass spectrometry ( $\mathrm{MS}^{2}$ ) experiments. In all cases, dissociation starts with homolytic cleavages in the polymer chain. The incipient radicals generated from linear structures unzip extensively by a combination of monomer losses and backbiting rearrangements; as a result, low-mass dissociation products dominate. The incipient radicals generated from macrocyclic structures, on the other hand, remain in the same molecule and in close proximity. This favors disproportionation, viz. intramolecular $\mathrm{H}^{*}$ transfer between the radicals, at the expense of fragmentation via consecutive monomer losses, thus leading to abundant high-mass fragments.

The unique fragmentation reactivities of linear and cyclic polystyrenes and polybutadienes allow for unambiguous determination of the correct architecture. Architectural assignment is possible by simple visual inspection of relative fragment intensity patterns. Rigorous interpretation of the fragment peaks is, however, essential for identifying specific substituents or linkers in the analyzed molecules. It is worth noting that the molecules investigated decompose via free radical chemistry processes, which lead to fragment ion distributions that reflect the polymer architecture. Other polymers fragmenting via analogous free radical chemistry, such as poly(methyl acrylate)s and poly(methyl methacrylate)s, are expected to show a similar dependence of fragment ion distribution on architecture.

\section{Acknowledgments}

The authors thank the National Science Foundation (CHE0517909, CBET-0730692, and CHE-1012636) for generous financial support, and Dr. Vijay S. Chavan for the polybutadiene samples.

\section{References}

1. Pethrick, R.A., Petkov, P., Zaikov, G.E., Rakovsky, S.K. (eds.): Monomers, Oligomers, Polymers, Composites, and Nanocomposites Research: Synthesis, Properties, and Applications. Nova Science Publishers, Inc., Hauppauge, NY (2010)

2. Kricheldorf, H.R.: Cyclic polymers: Synthetic strategies and physical properties. J. Polym. Sci. Part A: Polym. Chem. 48, 251-284 (2010)

3. Schappacher, M., Deffieux, A.: $\alpha$-Acetal- $\omega$-bis(hydroxymethyl) heterodifunctional polystyrene: Synthesis, characterization, and investigation of intramolecular end-to-end ring closure. Macromolecules 34, 5827$5832(2001)$

4. Laurent, B.A., Grayson, S.M.: An efficient route to well-defined macrocyclic polymers via "click" cyclization. J. Am. Chem. Soc. 128, 4238-4239 (2006)

5. Endo, K.: Synthesis and properties of cyclic polymers. Adv. Polym. Sci. 217, 121-183 (2008)

6. Laurent, B.A., Grayson, S.M.: Synthetic approaches for the preparation of cyclic polymers. Chem. Soc. Rev. 38, 2202-2213 (2009)

7. Hoskins, J.N., Grayson, S.M.: Synthesis and degradation behavior of cyclic poly(e-caprolactone). Macromolecules 42, 6406-6413 (2009)

8. Quirk, R.P., Wang, S.-F., Foster, M.D., Wesdemiotis, C., Yol, A.M.: Synthesis of cyclic polystyrenes using living anionic polymerization and metathesis ring-closure. Macromolecules 44, 7538-7545 (2011)

9. Hoskins, J.N., Trimpin, S., Grayson, S.M.: Architectural differentiation of linear and cyclic polymeric isomers by ion mobility spectrometrymass spectrometry. Macromolecules 44, 6915-6918 (2011)

10. Adhiya, A., Wesdemiotis, C.: Poly(propylene imine) dendrimer conformations in the gas phase: A tandem mass spectrometry study. Int. J. Mass Spectrom. 214, 75-88 (2002)

11. Adamus, G., Montaudo, M.S., Montaudo, G., Kowalczuk, M.: Molecular architecture of poly[(R, S)-3-hydroxybutyrate-co-6-hydroxyhexanoate] and poly[(R, S)-3-hydroxybutyrate-co-(R, S)-2-hydroxyhexanoate] oligomers investigated by electrospray ionization ion-trap multistage mass spectrometry. Rapid Commun. Mass Spectrom. 18, 1436-1446 (2004)

12. Wollyung, K.M., Wesdemiotis, C., Nagy, A., Kennedy, J.P.: Synthesis and mass spectrometry characterization of centrally and terminally amine-functionalized polyisobutylenes. J. Polym. Sci. A: Polym. Chem. 43, 946-958 (2004)

13. Giguere, M.-S., Mayer, P.M.: Climbing the internal energy ladder: The unimolecular decomposition of ionized poly(vinyl acetate). Int. J. Mass Spectrom. 231, 59-68 (2004)

14. Casey, J., Alhazmi, A., Mayer, P.M.: Conformation effects on the dissociation of ionized polymers. Eur. J. Mass Spectrom. 11, 557-563 (2005)

15. Rizzarelli, P., Puglisi, C., Montaudo, G.: Matrix-assisted laser desorption/ionization time-of-flight/time-of-flight tandem mass spectra of poly (butylenes adipate). Rapid Commun. Mass Spectrom. 20, 1683-1694 (2006)

16. Gies, A.P., Vergne, M.J., Orndorff, R.L., Hercules, D.M.: MALDITOF/TOF CID study of polystyrene fragmentation reactions. Macromolecules 40, 7493-7504 (2007)

17. Jackson, A.T., Green, M.R., Bateman, R.H.: Generation of end-group information from polyethers by matrix-assisted laser desorption/ionization collision-induced dissociation mass spectrometry. Rapid Commun. Mass Spectrom. 20, 3542-3550 (2008)

18. Jackson, A.T., Robertson, D.F.: Chain end characterization. Compr. Anal. Chem. 53, 171-203 (2008)

19. Rizzarelli, P., Puglisi, C.: Structural characterization of synthetic poly (ester amide) from sebacic acid and 4-amino-1-butanol by matrixassisted laser desorption ionization time-of-flight/time-of-flight tandem mass spectrometry. Rapid Commun. Mass Spectrom. 22, 739-754 (2008)

20. Polce, M.J., Ocampo, M., Quirk, R.P., Wesdemiotis, C.: Tandem mass spectrometry characteristics of silver-cationized polystyrenes: Backbone degradation via free radical chemistry. Anal. Chem. 80, 347-354 (2008)

21. Polce, M.J., Ocampo, M., Quirk, R.P., Leigh, A.M., Wesdemiotis, C.: Tandem mass spectrometry characteristics of silver-cationized polystyrenes: Internal energy, size, and chain end versus backbone substituent effects. Anal. Chem. 80, 355-362 (2008)

22. Chaicharoen, K., Polce, M.J., Singh, A., Pugh, C.: Characterization of linear and branched polyacrylates by tandem mass spectrometry. Anal. Bioanal. Chem. 392, 595-607 (2008) 
23. Gies, A.P., Vergne, M.J., Orndorff, R.L., Hercules, D.M.: MALDITOF/TOF CID study of 4-alkyl-substituted polystyrene fragmentation reactions. Anal. Bioanal. Chem. 392, 609-626 (2008)

24. Girod, M., Phan, T.N.T., Charles, L.: Microstructural study of a nitroxide-mediated poly(ethylene oxide)/polystyrene block copolymer (PEO-b-PS) by electrospray tandem mass spectrometry. J. Am. Soc. Mass Spectrom. 19, 1163-1175 (2008)

25. Ellison, S.T., Gies, A.P., Hercules, D.M., Morgan, S.L.: Py-GC/MS and MALDI-TOF/TOF CID study of polysulfone fragmentation reactions. Macromolecules 42, 3005-3013 (2009)

26. Giordanengo, R., Viel, S., Allard-Breton, B., Thevand, A., Charles, L.: Tandem mass spectrometry of poly(methacrylic acid) oligomers produced by negative mode electrospray ionization. J. Am. Soc. Mass Spectrom. 20, 25-33 (2009)

27. Giordanengo, R., Viel, S., Allard-Breton, B., Thevand, A., Charles, L.: Positive mode electrospray tandem mass spectrometry of poly(methacrylic acid) oligomers. Rapid Commun. Mass Spectrom. 23, 1557-1562 (2009)

28. Alhazmi, A.M., Mayer, P.M.: Protonating polymer oligomers in the gas phase to change fragmentation pathways. J. Am. Soc. Mass Spectrom. 20, 60-66 (2009)

29. Renaud, J., Alhazmi, A.M., Mayer, P.M.: Comparing the fragmentation chemistry of gas-phase adducts of poly(dimethylsiloxane) oligomers with metal and organic ions. Can. J. Chem. 87, 453-459 (2009)

30. Gies, A.P., Geibel, J.F., Hercules, D.M.: MALDI-TOF/TOF CID study of poly(p-phenylene sulfide) fragmentation reactions. Macromolecules 43, 952-967 (2010)

31. Knop, K., Jahn, B.O., Hager, M.D., Crecelius, A., Gottschaldt, M., Schubert, U.S.: Systematic MALDI-TOF CID investigation on different substituted mPEG 2000. Macromol. Chem. Phys. 211, 677-684 (2010)

32. Giordanengo, R., Viel, S., Hidalgo, M., Allard-Breton, B., Thevand, A., Charles, L.: Methylation of acidic moieties in poly(methyl methacrylate-co-methacrylic acid) copolymers for end-group characterization by tandem mass spectrometry. Rapid Commun. Mass Spectrom. 24, 19411947 (2010)

33. Tintaru, A., Monnier, V., Bouillon, C., Giordanengo, R., Quelever, G., Peng, L., Charles, L.: Structural characterization of poly(amino)ester dendrimers and related impurities by electrospray tandem mass spectrometry. Rapid Commun. Mass Spectrom. 24, 2207-2216 (2010)

34. Kuki, A., Nagy, L., Memboeuf, A., Drahos, L., Vekey, K., Zsuga, M., Keki, S.: Energy-dependent collision-induced dissociation of lithiated polytetrahydrofuran: Effect of the size on the fragmentation properties. J. Am. Soc. Mass Spectrom. 21, 1753-1761 (2010)

35. Memboeuf, A., Nasioudis, A., Indelicato, S., Pollreisz, F., Kuki, A., Keki, S., van den Brink, O.F., Vekey, K., Drahos, L.: Size effect on fragmentation in tandem mass spectrometry. Anal. Chem. 82, 2294-2302 (2010)

36. Nasioudis, A., Memboeuf, A., Heeren, R.M.A., Smith, D.F., Vekey, K., Drahos, L., van den Brink, O.F.: Discrimination of polymers by using their characteristic collision energy in tandem mass spectrometry. Anal. Chem. 82, 9350-9356 (2010)
37. Fouquet, T., Humbel, S., Charles, L.: Tandem mass spectrometry of trimethylsilyl-terminated poly (dimethylsiloxane) ammonium adducts generated by electrospray ionization. J. Am. Soc. Mass Spectrom. 22, 649-658 (2011)

38. Nasioudis, A., Heeren, R.M.A., van Doormalen, I., de Wijs-Rot, N., van den Brink, O.F.: Electrospray ionization tandem mass spectrometry of ammonium cationized polyethers. J. Am. Soc. Mass Spectrom. 22, 837844 (2011)

39. Fouquet, T., Phan, T.N.T., Charles, L.: Tandem mass spectrometry of electrosprayed polyhedral oligomeric silsesquioxane compounds with different substituents. Rapid Commun. Mass Spectrom. 26, 765-774 (2012)

40. Tanaka, K., Waki, H., Ido, Y., Akita, S., Yoshida, Y., Yoshida, T.: Protein and polymer analyses up to $\mathrm{m} / \mathrm{z} 100,000$ by laser ionization time-of-flight mass spectrometry. Rapid Commun. Mass Spec. 2, 151153 (1988)

41. Karas, M., Hillenkamp, F.: Laser desorption ionization of proteins with molecular masses exceeding 10,000 daltons. Anal. Chem. 60, 22992301 (1988)

42. Wesdemiotis, C., Solak, N., Polce, M.J., Dabney, D.E., Chaicharoen, K., Katzenmeyer, B.C.: Fragmentation pathways of polymer ions. Mass Spectrom. Rev. 30, 523-559 (2011)

43. Suckau, D., Reseman, A., Schuerenberg, M., Hufnagel, P., Franzen, J., Holle, A.: A novel MALDI LIFT-TOF/TOF mass spectrometer for proteomics. Anal. Bioanal. Chem. 376, 952-965 (2003)

44. Scionti, V., Wesdemiotis, C.: Tandem mass spectrometry analysis of polymer structures and architectures. In: Barner-Kowollik, C., Gruendling, T., Falkenhagen, J., Weidner, S. (eds.) Mass Spectrometry in Polymer Chemistry, Chap. 3, pp. 57-84. Wiley-VCH, Weinheim, Germany (2011)

45. Wang, S.-F.: Synthesis and characterization of surface relaxations of macrocyclic polystyrenes and interfacial segregation in blends with linear polystyrenes. Ph.D. Dissertation, University of Akron (2011)

46. Chavan, V.S.: Controlled synthesis and characterization of branched, functionalized, and cyclic polymers. Ph.D. Dissertation, University of Akron (2011)

47. Bielawski, C.W., Benitez, D., Grubbs, R.H.: Synthesis of cyclic polybutadiene via ring-opening metathesis polymerization: The importance of removing trace linear contaminants. J. Am. Chem. Soc. 125, 8424-8425 (2003)

48. McMillen, D.F., Golden, D.M.: Hydrocarbon bond dissociation energies. Ann. Rev. Phys. Chem. 33, 493-532 (1982)

49. Lide, D.R. (ed.). CRC Handbook of Chemistry and Physics, 89th ed. CRC Press: Boca Raton, FL, pp. 9-70 and 9-74 (2008)

50. Dabney, D.E.: Analysis of synthetic polymers by mass spectrometry and tandem mass spectrometry. Ph.D. Dissertation, University of Akron (2009)

51. Susteric, Z.: Thermal activation/free volume-approach to elastomeric tearing. Kautschuk Gummi Kunststoffe 45, 480-486 (1992) 